\title{
Consumo de alimentos ultraprocessados e fatores associados no primeiro ano de vida em Cruzeiro do Sul, Acre, Brasil
}

\author{
Consumption of ultra-processed foods during the first year of life \\ and associated factors in Cruzeiro do Sul, Brazil
}

Mariana Bossi Nogueira (https://orcid.org/0000-0001-7017-8718) ${ }^{1}$
Lalucha Mazzucchetti (https://orcid.org/0000-0002-9649-5727) ${ }^{1}$
Paola Soledad Mosquera (https://orcid.org/0000-0001-8423-7344) ${ }^{1}$
Marly Augusto Cardoso (https://orcid.org/0000-0003-0973-3908) ${ }^{1}$
Maíra Barreto Malta (https://orcid.org/0000-0003-4993-1589) ${ }^{2}$
${ }^{1}$ Faculdade de Saúde Pública, Universidade de São Paulo. Av. Dr. Arnaldo 715, Cerqueira César. 01246 904 São Paulo SP Brasil. maaribnogueira@gmail.com

${ }^{2}$ Pós-Graduação em Saúde Coletiva, Universidade Católica de Santos. Santos SP Brasil.

\begin{abstract}
This article aims to assess the consumption of ultra-processed foods (UPFs) during the first year of life and associated factors. We analyzed data from the one-year follow-up of the MINA-Brazil birth cohort. Socioeconomic, demographic and obstetric data were collected in the baseline study and at one-year follow-up ( $n$ =774). Dietary intake during the previous day was assessed using a food frequency questionnaire to determine UPF consumption. Multiple Poisson regression with robust variance was performed to estimate prevalence ratios $(P R)$ and confidence intervals (95\%CI) for factors associated with the consumption of $\geq 3$ UPFs. Prevalence of UPF consumption and consumption of $\geq 3$ UPFs was $87.5 \%$ and $40.5 \%$, respectively. Consumption of $\geq 3$ UPFs was associated with lower maternal education ( $\leq 9$ years of schooling [PR: 2.02 95\%CI 1.42; 2.87] and between 10-12 years of schooling [PR: 1.55, 95\%CI 1, 11; 2.14]), below-average wealth index (PR: 1.26, 95\%CI 1.04; 1.53), and having a teenage mother (PR:1.19, 95\%CI 1.00; 1.49). Lack of dietary diversity was inversely associated with the outcome (RP: 0,65, IC95\% 0,51; 0,81 ). The consumption of $\geq 3$ UPFs was associated with lower wealth index, education and maternal age. Dietary diversity was associated with higher levels of UPF consumption.
\end{abstract}

Key words Child nutrition, Industrialized foods, Complementary foods, Cohort study
Resumo $O$ objetivo deste artigo é avaliar o consumo de alimentos ultraprocessados (AUP) e fatores associados no primeiro ano de vida. Trata-se da análise de dados do seguimento de um ano da coorte de nascimentos MINA-Brasil. Foram coletados dados socioeconômicos, demográficos e obstétricos na maternidade e no seguimento de um ano $(n=774)$, investigando consumo alimentar do dia anterior à entrevista, a fim de avaliar o consumo de AUP. A razão de prevalência $(R P) e$ o intervalo de confianças (IC 95\%) para consumo $\geq 3$ AUP foram estimados em modelo de regressão múltiplo de Poisson com variância robusta. A prevalência de consumo $\geq 1$ AUP foi $87,5 \%$; $e \geq 3$ AUP de 40,5\%, estando associado a: menor escolaridade materna ( $\leq 9$ anos [ $R P$ : 1,97 IC 95\% 1,38; 2,80] e entre 10-12 anos [RP: 1,58, IC 95\% 1,13; 2,20) comparados com $>12$ anos de escolaridade, indice de riqueza abaixo da média ( $R P$ : 1,26, IC $95 \% 1,04 ; 1,53)$ e ter mãe adolescente (RP: 1,28, IC 95\% 1,06; 1,55). A ausência de diversidade da dieta foi inversamente associada ao desfecho (RP: 0,65, IC 95\% 0,51;0,81). O consumo $\geq 3$ AUP se associou às características maternas de menores escolaridade, indice de riqueza e idade materna, $e$ a diversidade da dieta ao maior consumo de AUP. Palavras-chave Alimentação infantil, Alimento industrializado, Alimentação complementar, Estudo de coorte 


\section{Introdução}

Os primeiros mil dias de vida de um indivíduo são caracterizados por um intenso processo de crescimento e maturação que constitui uma importante janela de oportunidades para promoção da alimentação e nutrição saudáveis. Nesse período, as escolhas alimentares do binômio mãe-bebê são determinantes para o perfil de crescimento e desenvolvimento neurocognitivo plenos, bem como para a redução dos riscos de diversas doenças e morbidades que terão repercussão ao longo de toda a vida ${ }^{1}$.

Dada a importância desse período, a Organização Mundial da Saúde (OMS) recomenda o aleitamento materno exclusivo até os seis meses de vida, e complementado até os dois anos ou mais ${ }^{2}$. Recomenda-se ainda que a alimentação complementar, iniciada a partir de seis meses, seja adequada e segura, não sendo ofertados alimentos ultraprocessados (AUP) nos dois primeiros anos, uma vez que geralmente são ricos em sódio, açucares e gorduras prejudiciais à saúde, além do baixo teor de fibras, vitaminas e minerais ${ }^{3}$. Define-se alimentos ultraprocessados (AUP) como "formulações de ingredientes industriais contendo pouco ou nenhum alimento intacto"4.

Sabe-se da importância dos primeiros anos de vida de um indivíduo no que diz respeito à formação de seus hábitos alimentares. Estudos apontam que os alimentos consumidos por crianças com seis anos de idade coincidem com aqueles ingeridos por elas no primeiro ano de vida, denotando que hábitos alimentares não saudáveis nesse período repercutem ao longo da vida ${ }^{5}$. Além disso, o consumo de AUP está atrelado ao aumento da epidemia de obesidade, diabetes e outras doenças crônicas não-transmissíveis $^{3}$. Revisão sistemática que incluiu 26 estudos concluiu haver associações positivas entre o consumo de AUP e a gordura corporal na infância e adolescência ${ }^{6}$. O alto consumo de AUP, contudo, já é uma realidade. Relatório produzido pela Organização Pan Americana de Saúde (OPAS) ${ }^{7}$ constatou aumento de $48 \%$ na circulação/venda dos AUP entre os anos 2000 e 2013 em 13 países latino-americanos, evidenciando o atual contexto de modificação de hábitos e padrões alimentares onde as dietas de alta densidade energética ganham destaque ${ }^{8}$.

Apesar de a maioria dos estudos sobre consumo de AUP ter sido realizado com adolescentes e adultos, estudo transversal brasileiro com pré-escolares identificou que a idade média de introdução de AUP na dieta foi de seis meses de vida9. Além disso, a 2a Pesquisa de Prevalência de Aleitamento Materno, conduzida nas capitais brasileiras e no Distrito Federal em 2009, já apontava um consumo de $11,6 \%$ de bebidas açucaradas e de $71,7 \%$ de bolachas e/ou salgadinhos por crianças com idade entre 9 e 12 meses $^{10}$. Outro estudo transversal de base populacional, utilizando dados da Pesquisa Nacional de Saúde (PNDS), apontou que 72,6\% de crianças entre 6 e 12 meses consumiram "alimentos não saudáveis" (refrigerantes, sucos artificiais, bolachas e doces), considerados no estudo como proxy de AUP ${ }^{11}$. Poucos estudos investigaram os fatores associados ao consumo de AUP nesse período da vida. A escolaridade materna e o tempo entre o nascimento e a primeira consulta da criança em unidade de saúde foram apontados em estudo transversal realizado em Embu das Artes, Brasil, com 181 crianças com idades entre 6 e 12 meses $^{12}$.

Considerando-se a essencialidade das práticas alimentares infantis saudáveis, a elevação do consumo de AUP na infância, bem como a carência de estudos sobre consumo de AUP no primeiro ano de vida, o presente estudo teve como objetivo avaliar esse consumo e fatores associados no primeiro ano de vida em município da Amazônia Ocidental Brasileira.

\section{Métodos}

Trata-se de um estudo longitudinal de base populacional com dados obtidos da Coorte de Nascimentos MINA-Brasil: Saúde e Nutrição Materno-Infantil em Cruzeiro do Sul, Acre. Na linha de base, o recrutamento do binômio mãe-bebê foi realizado na única maternidade do município, entre julho de 2015 e junho de 2016, conforme descrição em publicação anterior ${ }^{13}$. Todas as mães internadas para parto, residentes em Cruzeiro do Sul, estado do Acre, foram convidadas a participar do estudo.

Após concordância por escrito, as mães responderam a um formulário estruturado sobre questões socioeconômicas, demográficas e histórico de saúde. Durante o período de recrutamento das participantes na maternidade, registraram-se 1.881 internações para parto. Dessas, $128(6,9 \%)$ foram consideradas não elegíveis, sendo 112 abortos e 16 natimortos, totalizando 1.753 nascidos vivos, 184 mães dos nascidos vivos se recusaram a participar do estudo e 18 não foram contatadas pela equipe de pesquisa. Entre as 1.551 mães contatadas, 305 não foram inclu- 
ídas por residirem em área rural. Para o seguimento de um ano de idade, 25 (2,0\% dos 1246 elegíveis) se recusaram a participar dessa fase do estudo, quatro crianças faleceram, 38 se mudaram para a área rural ou outros municípios, 110 não compareceram à avaliação após três tentativas de agendamento e 285 não foram localizadas, de forma que foram acompanhadas 774 crianças $(63,2 \% \text { das elegíveis })^{13}$.

$\mathrm{Na}$ maternidade, uma equipe de pesquisa previamente treinada entrevistou as puérperas, sendo coletados dados maternos como: data de nascimento para cálculo da idade materna, categorizada em (< 19 anos, 19-34, $\geq 35$; e em adolescente: sim, não $)^{14}$, cor da pele autorreferida (branco, pardo/preto/amarelo/indígena), escolaridade $(0-4,5-8,9-11, \geq 12$ anos completos de estudo), recebimento do auxílio Bolsa Família ( $\operatorname{sim}$, não), mãe exerce atividade remunerada ( $\operatorname{sim}$, não), vive com companheiro ( $\operatorname{sim}$, não), mãe se autodeclarar chefe do domicílio (sim, não), primigesta ( $\operatorname{sim}$, não) e índice de riqueza (abaixo da média, acima da média).

$\mathrm{O}$ índice de riqueza foi utilizado como proxy do status socioeconômico familiar ${ }^{15}$, e foi construído pela análise dos componentes principais, derivados dos seguintes bens domésticos: televisão, aparelho de som, computador, aparelho de DVD, internet, TV a cabo, geladeira, fogão a gás, liquidificador, micro-ondas, ferro elétrico, sofá, telefone fixo, celular, bicicleta, motocicleta, carro, posse de terra e criação de gado. Os escores gerados foram então somados e categorizados em acima ou abaixo da média. Maior detalhamento do método utilizado para essas análises na Coorte MINA-Brasil foi publicado anteriormente ${ }^{13}$.

Durante a entrevista, informações sobre o número total de consultas de pré-natal foram obtidas da caderneta de pré-natal da mãe, e categorizadas de acordo com as recomendações do Ministério da Saúde ${ }^{16}$ ( $<6, \geq 6$ consultas). Dos prontuários hospitalares foram coletadas as seguintes informações: sexo da criança (feminino, masculino), peso ao nascer em gramas, posteriormente categorizado (baixo peso $<2.500$, peso adequado 2.500-3.999, ou macrossomia 4.000) e idade gestacional em semanas completas. Trinta e quatro porcento das mães tiveram estimativa de idade gestacional confirmada por ultrassom realizado pela equipe MINA-Brasil, com uma diferença média aceitável em comparação com os registros hospitalares, conforme descrito em publicação anterior ${ }^{17}$.

Quando as crianças completaram um ano de vida, membros da equipe de pesquisa entra- ram em contato, via telefone, com as mães para agendamento de uma avaliação presencial, realizada em unidades básicas de saúde. Realizouse avaliação antropométrica das crianças, além de aplicação de questionário relativo às práticas alimentares gerais na primeira infância ${ }^{18}$ : uso de mamadeiras (sim, não) e chupetas (sim, não), aleitamento materno no seguimento de um ano (sim, não) e aleitamento materno exclusivo ou predominante aos seis meses de vida ( $\operatorname{sim}$, não).

A coleta dos dados antropométricos foi feita em duplicata por integrantes da equipe de pesquisa devidamente treinados e seguiu procedimentos padronizados, com equipamentos devidamente calibrados ${ }^{19}$. Para as medidas de peso, foi utilizada balança portátil digital eletrônica, da marca Tanita, com capacidade de $150 \mathrm{~kg}$ e variação de $0,1 \mathrm{~kg}$, e o comprimento foi obtido em infantômetro portátil. O estado nutricional das crianças no primeiro ano de vida foi realizado segundo cálculo de IMC para idade, sendo o escore $Z>+2$ considerado como excesso de peso, segundo padrão de referência da $\mathrm{OMS}^{14}$. Para análise do comprimento para a idade, foram consideradas com déficit de comprimento para a idade as crianças que apresentaram escore $Z<-2^{14}$.

Para classificação antropométrica materna no período pré-gestacional, utilizou-se peso pré-gestacional autorreferido. Para mães com idade maior ou igual a 19 anos, foram calculados o IMC e o estado nutricional classificado segundo critérios da OMS (1995) ${ }^{19}$ : baixo peso (IMC $<18,5 \mathrm{~kg} / \mathrm{m}^{2}$ ); peso adequado (IMC 18,5$24,9 \mathrm{~kg} / \mathrm{m}^{2}$ ); sobrepeso (IMC 25,0-29,9 kg/m²) e obesidade ( $\geq 30 \mathrm{~kg} / \mathrm{m}^{2}$ ). Já para as mães com idade menor do que 19 anos, a definição do estado nutricional pré-gestacional foi realizada com auxílio do programa WHO Anthro Plus (2009), que calcula IMC em escore $\mathrm{Z}$ para idade em adolescentes, considerando-se os seguintes pontos de corte para sua classificação: baixo peso (escore Z $<-2$ ); peso adequado (escore $Z-2$ a $<1$ ); sobrepeso (escore $Z \geq+1$ ) e obesidade (escore $Z \geq+2$ ).

A avaliação do consumo alimentar das crianças foi realizada mediante questionário de frequência alimentar estruturado referente ao consumo de alimentos e bebidas no dia anterior à entrevista, quando esse representou um dia habitual de consumo da criança. Para cada um dos 23 alimentos/grupos de alimentos questionados, e para a opção "outro alimento", havia nove categorias de respostas (não consumiu, ao acordar, meio da manhã, almoço, meio da tarde, jantar, antes de dormir, madrugada, não sabe), podendo ser assinalados mais do que uma opção. A 
lista de alimentos foi adaptada de questionário elaborado por Oliveira e colaboradores ${ }^{18}$, sendo inseridos os alimentos regionais selecionados segundo questionário utilizado por Castro e colaboradores em estudo amazônico ${ }^{20}$. Para os casos em que o dia anterior à entrevista não foi um dia de consumo alimentar habitual, foi solicitado à mãe ou cuidador que relatasse o último dia de alimentação habitual da criança. Em vista disso, foi aplicada a seguinte questão: "No dia de ontem, a alimentação da criança foi parecida com o que ela costuma comer?"

Os AUP incluídos no questionário aplicado foram: iogurte industrializado, suco artificial, refrigerante, guloseimas, biscoito, salgadinho de pacote, salsicha e macarrão instantâneo. Além desses, outros AUP mencionados durante a entrevista em pergunta aberta sobre consumo de "outros alimentos" também foram considerados (achocolatado, sorvete, gelatina, bolo e sopa industrializados). Para a estimativa da prevalência do consumo de AUP, considerou-se a ingestão de pelo menos um alimento dessa categoria no dia anterior à entrevista. Dada a elevada prevalência encontrada de consumo de um AUP no dia anterior à entrevista, comparada à recomendação do Guia alimentar para crianças brasileiras menores de dois anos ${ }^{4}$ (de que não sejam ofertados AUP nessa fase da vida), optou-se por investigar os fatores associados ao consumo de três ou mais AUP.

A diversidade da dieta foi classificada segundo indicador de diversidade da alimentação complementar proposto pela $\mathrm{WHO}^{21}$, definido pelo consumo de ao menos quatro dos sete grupos alimentares recomendados (grãos, raízes e tubérculos; leguminosas e oleaginosas; produtos lácteos; carnes; ovos; frutas e hortaliças ricas em vitamina A, e outras frutas e hortaliças).

As características socioeconômicas, demográficas, obstétricas e perinatais das crianças acompanhadas no seguimento de um ano foram comparadas às perdas de seguimento pelo teste qui-quadrado. Quanto à descrição da população estudada, as variáveis discretas foram apresentadas em medidas de tendência central e dispersão (média e desvio-padrão - dp) e as variáveis categóricas foram apresentadas em frequências totais e segundo número de AUP consumidos no dia anterior à entrevista (não consumiu; consumiu de um a dois AUP; consumiu três ou mais AUP). As categorias também foram comparadas mediante teste qui-quadrado.

A razão de prevalência ( $\mathrm{RP}$ ) e o intervalo de confianças (IC 95\%) para consumo de um AUP e três ou mais AUP no seguimento de um ano da coorte foram estimados em modelo de regressão de Poisson com variância robusta. Os modelos de regressão múltiplos foram baseados em uma estrutura conceitual hierárquica ${ }^{22}$ considerando as associações com exposições em níveis distal (fatores socioeconômicos e demográficos), intermediário (fatores obstétricos e perinatais) e proximal (práticas alimentares e estado nutricional no seguimento de um ano). A análise bruta para cada variável independente foi realizada retendo aquelas associadas ao desfecho em $\mathrm{p}<0,20$. Em cada nível de determinação, as variáveis associadas ao desfecho em $\mathrm{p}<0,10$ foram mantidas no modelo múltiplo final. As análises dos dados foram realizadas com auxílio do pacote estatístico Stata 14.0, ao nível de significância de $\mathrm{p}<0,05$.

O Termo de Consentimento Livre e Esclarecido foi assinado pelas mães após explicações sobre o objetivo da pesquisa e a garantia de sigilo das informações. Para as mães adolescentes, o consentimento foi dado pelo responsável. O projeto foi aprovado pelo Comitê de Ética em Pesquisa Faculdade de Saúde Pública da Universidade de São Paulo (FSP/USP parecer no 872.613).

\section{Resultados}

No presente estudo foram avaliadas 774 crianças não gemelares, residentes em área urbana do município de Cruzeiro do Sul, Acre. Considerando as crianças acompanhadas no seguimento de um ano da coorte em relação aos não acompanhados ( $\mathrm{n}=450)$, observou-se que não houve diferença em relação à cor da pele materna autorreferida, à situação conjugal (viver ou não com companheiro) e ao fato de a mãe ser chefe de domicílio. Houve diferença significativa em termos estatísticos, com menores proporções de participantes acompanhados em relação aos não acompanhados, respectivamente, entre mães com idade $<19$ anos $(16,0 \%$ versus $23,3 \% ; p=0,002)$, com $\leq 9$ anos de escolaridade $(29,7 \%$ versus $45,7 \%$; $\mathrm{p}<$ $0,0001)$ e abaixo da média do índice de riqueza ( $45,5 \%$ versus $58,7 \%$; $p<0,0001)$ na linha de base do estudo. Quanto às características obstétricas e perinatais, não houve diferenças estatisticamente significantes para primiparidade, sexo e peso do bebê ao nascer.

No momento do parto, a idade média das mães foi de 25,5 anos ( $\mathrm{dp} \pm 6,6$ ), variando entre 14 e 43 anos, das quais $16 \%$ eram adolescentes e apenas $19,7 \%$ havia completado pelo menos 12 anos de estudo (no momento do parto). Além 
disso, grande parte $(36,9 \%)$ das mulheres recebia auxílio do Programa Bolsa Família (Tabela 1).

Em relação às crianças, $96 \%$ receberam aleitamento materno no primeiro dia de vida, aproximadamente $6 \%$ tinham baixo peso ao nascer e a mesma porcentagem apresentou macrossomia fetal. A média de idade das crianças avaliadas no seguimento do primeiro ano de vida foi de 12,7 meses $(\mathrm{dp} \pm 0,7)$. As frequências de déficit de comprimento para a idade e excesso de peso (IMC para idade) foram 2,2\% e 6,6\%, respectivamente.

No total, 87,5\% (IC 95\% 0,85; 0,90) das crianças consumiram pelo menos um AUP no dia anterior à entrevista, sendo que 47\% (IC 95\% $0,44 ; 0,51)$ consumiram um ou dois AUP e 40,5\% (IC95\% 0,37;0,44) três ou mais AUP. Biscoito, iogurte industrializado, guloseimas, salgadinho de pacote e suco artificial foram os alimentos que mais contribuíram para esse resultado em $66,4 \%$, $53,2 \%, 18,1 \%, 17,9 \%, 14,7 \%$, respectivamente (dados apresentados na Figura 1). Apenas 5\% das mães $(n=39)$ afirmaram que a alimentação da criança no dia anterior à entrevista não representou um dia de consumo alimentar habitual.

A Tabela 2 apresenta as análises brutas entre as exposições e o consumo de AUP no dia anterior à entrevista. Os fatores associados ao consumo de três ou mais AUP nas análises brutas foram: ter mãe adolescente, menor escolaridade materna, índice de riqueza abaixo da média, receber bolsa família, mãe não exercer atividade remunerada. A ausência de diversidade na dieta foi inversamente associada ao desfecho. A Tabela 3 apresenta os fatores associados ao consumo de três ou mais AUP no dia anterior à entrevista do seguimento de um ano. Após ajuste por sexo e idade da criança, as características associadas ao maior consumo de AUP foram: menor escolaridade materna $(\leq 9$ anos [RP: 1,97 ; IC 95\% 1,38; 2,80] e entre 10 e 12 anos de escolaridade [RP: 1,58; IC 95\% 1,13; $2,20]$ ), em comparação com aquelas com mais de 12 anos de escolaridade, índice de riqueza abaixo da média (RP: 1,26; IC 95\% 1,04; 1,53) e ser filho de mãe adolescente (RP: 1,28; IC 95\% 1,06; 1,55). Ausência de diversidade da alimentação complementar foi inversamente associado ao consumo excessivo de AUP (RP: 0,65; IC 95\% 0,51; 0,81).

\section{Discussão}

O presente estudo se destaca por ser o primeiro a investigar o consumo de AUP e fatores associados em crianças no seguimento de um ano de vida de uma coorte na Amazônia Ocidental Brasileira. Os resultados apontaram para uma elevada prevalência de consumo desse tipo de alimento, visto que $87,5 \%$ haviam consumido um ou mais AUP e 40,5\% das crianças ingeriram três ou mais AUP no dia anterior à entrevista. Os achados evidenciaram uma realidade alarmante, tendo em vista que o Guia alimentar para crianças brasileiras menores de dois anos ${ }^{4}$ (2019) preconiza que não seja ofertado AUP nessa fase da vida, alertando para a sua contribuição no crescente excesso de peso e obesidade infantil. Esse é um período fundamental do desenvolvimento, em que ocorrem diversas modificações fisiológicas, como a formação das papilas gustativas, determinantes para a preferência por alguns alimentos ${ }^{23}$.

Em relação à proporção de crianças com diversidade da dieta adequada neste estudo (76,6\%), é importante destacar que esse aspecto da alimentação complementar foi estimado a partir do indicador proposto pela OMS - cuja construção é composta por sete grupos de alimentos (grãos, raízes e tubérculos; leguminosas e oleaginosas; produtos lácteos; carnes; ovos; frutas e hortaliças ricas em vitamina A, e outras frutas e hortaliças) e considera que a alimentação da criança é variada quando consome pelos menos quatro desses grupos ${ }^{21}$. Portanto, crianças que consumiram apenas alimentos dos grupos de carnes, ovos, grãos e lácteos, por exemplo, podem ter sido classificadas, inapropriadamente, como tendo uma alimentação variada ${ }^{18}$.

Ainda em relação à composição do indicador proposto pela OMS, deve-se notar que o mesmo não diferencia o grau de processamento dos alimentos, portanto não penaliza o consumo de $\mathrm{AUP}^{21}$, apesar das evidências sobre eventos adversos para a saúde infantil relacionados ao consumo de AUP ${ }^{4}$. Por exemplo, segundo o indicador de diversidade da OMS, o consumo de hambúrguer é computado no grupo das carnes, embora seja um AUP. Assim, a frequência observada para diversidade da dieta adequada neste estudo pode ter sido superestimada, contribuindo para a associação positiva entre diversidade da alimentação e consumo excessivo de AUP. Os nossos resultados apontam para a necessidade de estabelecimento de indicadores de alimentação complementar que considerem as recomendações do Guia alimentar brasileiro para crianças menores de dois anos ${ }^{4}$, incluindo como um indicador de alimentação complementar a avaliação do consumo de AUP, a fim de conseguir um monitoramento adeuado das práticas alimentares infantis.

Relvas e colaboradores ${ }^{12}$, em estudo transver- 
Tabela 1. Frequência (\%) de consumo de alimentos ultraprocessados (AUP) no primeiro ano de vida segundo características socioeconômicas, demográficas e obstétricas. Coorte MINA-Brasil, Cruzeiro do Sul, Acre, 20162017.

\begin{tabular}{|c|c|c|c|c|c|}
\hline \multirow[b]{2}{*}{ Variáveis } & \multicolumn{5}{|c|}{ Consumo de AUP no dia anterior à entrevista } \\
\hline & $\begin{array}{c}\text { Total } \\
(\mathrm{n}=774)\end{array}$ & $\begin{array}{c}\text { Não } \\
\text { consumiu } \\
(\mathbf{n}=97)\end{array}$ & $\begin{array}{l}\text { Consumiu } \\
1 \text { a } 2 \text { AUP } \\
(n=364) \\
\end{array}$ & $\begin{array}{c}\text { Consumiu } \\
3 \text { ou + AUP } \\
(n=313)\end{array}$ & $\mathbf{p}^{\mathrm{e}}$ \\
\hline Idade materna $(\mathrm{n}=774)$ & & & & & $<0,001$ \\
\hline$<19$ anos & 16,0 & 6,2 & 13,2 & 22,4 & \\
\hline $19-35$ anos & 73,4 & 75,3 & 75,3 & 70,6 & \\
\hline$\geq 35$ anos & 10,6 & 18,6 & 11,5 & 7,0 & \\
\hline Escolaridade materna $(\mathrm{n}=761)$ & & & & $<0,001$ & \\
\hline 0-4 anos de estudo & 7,2 & 6,2 & 5,1 & 10,1 & \\
\hline 5-8 anos de estudo & 13,0 & 12,4 & 9,8 & 16,9 & \\
\hline 9-11 anos de estudo & 51,7 & 45,4 & 50,0 & 55,5 & \\
\hline$\geq 12$ anos de estudo & 28,1 & 36,1 & 35,1 & 17,5 & \\
\hline Índice de riqueza $(\mathrm{n}=762)$ & & & & $<0,001$ & \\
\hline Abaixo da média & 50,0 & 38,1 & 44,8 & 59,7 & \\
\hline Acima da média & 50,0 & 61,9 & 55,2 & 40,3 & \\
\hline Recebe Bolsa Família (n=762) & 36,9 & 32,0 & 33,6 & 42,2 & 0,041 \\
\hline Cor da pele materna $(n=762)$ & & & & & 0,292 \\
\hline Branca & 12,2 & 8,3 & 14,0 & 11,4 & \\
\hline Negra/indígena/parda & 87,8 & 91,8 & 86,0 & 88,6 & \\
\hline Vive com companheiro $(\mathrm{n}=762)$ & 78,7 & 77,3 & 81,2 & 76,3 & 0,281 \\
\hline Mãe chefe do domicílio (n = 762) & 13,7 & 14,4 & 15,4 & 11,4 & 0,309 \\
\hline Primigesta $(\mathrm{n}=762)$ & 42,3 & 39,2 & 40,1 & 45,8 & 0,265 \\
\hline Número de consultas pré-natal $(\mathrm{n}=770)$ & & & & 0,463 & \\
\hline$<6$ consultas & 18,2 & 15,6 & 17,1 & 20,2 & \\
\hline$\geq 6$ consultas & 81,8 & 84,4 & 82,9 & 79,8 & \\
\hline Estado nutricional pré-gestacional $(\mathrm{n}=757)$ & & & & & 0,147 \\
\hline Peso adequado & 50,7 & 44,1 & 48,7 & 55,1 & \\
\hline Baixo peso & 4,2 & 2,2 & 3,6 & 5,5 & \\
\hline Sobrepeso & 30,9 & 38,7 & 31,9 & 27,4 & \\
\hline Obesidade & 14,1 & 15,1 & 15,7 & 12,1 & \\
\hline Sexo da criança $(\mathrm{n}=774)$ & & & & & 0,390 \\
\hline Feminino & 52,2 & 46,4 & 51,9 & 54,3 & \\
\hline Masculino & 47,8 & 53,6 & 48,1 & 45,7 & \\
\hline Peso ao nascer (gramas) $(\mathrm{n}=773)$ & & & & & 0,153 \\
\hline Baixo peso $(<2.500 \mathrm{~g})$ & 6,3 & 12,4 & 5,2 & 5,8 & \\
\hline Peso adequado (2.500-3.999 g) & 87,6 & 82,5 & 87,9 & 88,8 & \\
\hline Macrossomia $(\geq 4.000 \mathrm{~g})$ & 6,1 & 5,2 & 6,9 & 5,4 & \\
\hline Aleitamento materno no $1^{\circ}$ dia $(\mathrm{n}=774)$ & 96,0 & 95,9 & 95,6 & 96,5 & 0,843 \\
\hline Uso de mamadeiraa $(\mathrm{n}=774)$ & 62,7 & 69,1 & 62,6 & 60,7 & 0,330 \\
\hline Uso de chupetaa $(\mathrm{n}=774)$ & 22,0 & 30,9 & 20,6 & 20,8 & 0,074 \\
\hline $\begin{array}{l}\text { Aleitamento materno exclusivo ou predominante } \\
\text { até } 6 \text { meses }(n=628)\end{array}$ & 7,8 & 9,9 & 5,4 & 10,0 & 0,097 \\
\hline Aleitamento materno aos 12 meses $(\mathrm{n}=774)$ & 69,4 & 58,8 & 72,3 & 69,3 & 0,038 \\
\hline Déficit de comprimento para idade ${ }^{\mathrm{a} b},(\mathrm{n}=772)$ & 2,2 & 2,1 & 1,4 & 3,2 & 0,271 \\
\hline Excesso de pesoa, ${ }^{\mathrm{c}}(\mathrm{n}=772)$ & 6,6 & 7,2 & 8,3 & 4,5 & 0,127 \\
\hline Diversidade da dietaa, ${ }^{\mathrm{d}}(\mathrm{n}=774)$ & 76,6 & 66,0 & 74,3 & 82,1 & 0,002 \\
\hline
\end{tabular}




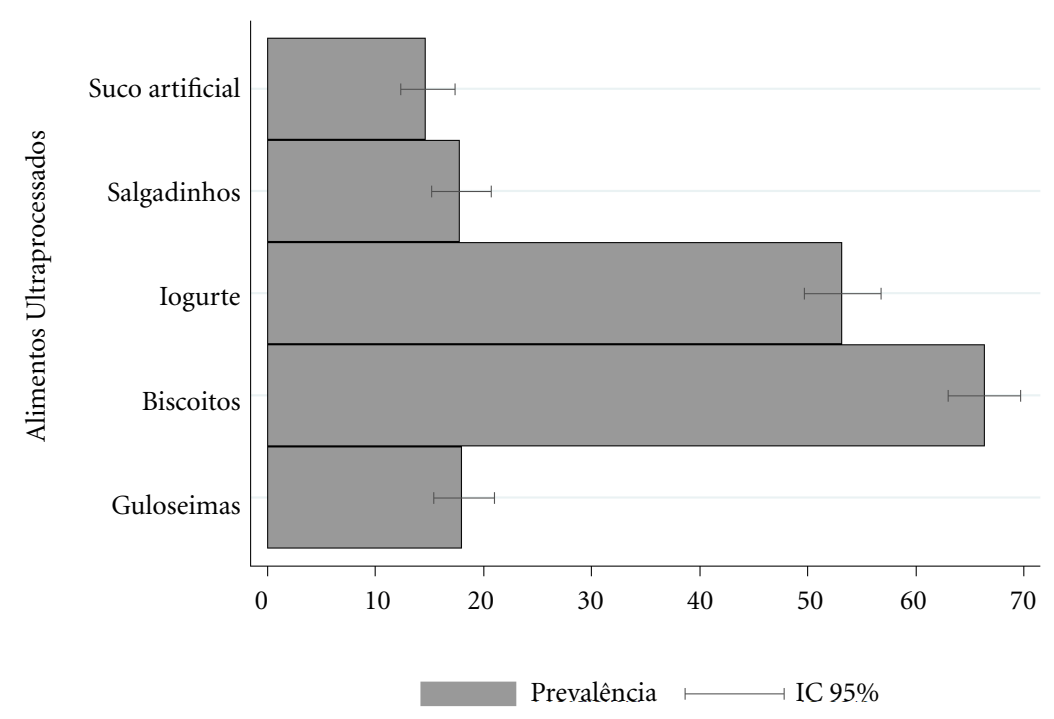

Figura 1. Prevalências e intervalo com 95\% de confiança (IC 95\%) dos alimentos ultraprocessados mais consumidos no primeiro ano de vida da Coorte MINA-Brasil, Cruzeiro do Sul, Acre.

Fonte: Autoras.

sal, avaliaram o consumo de AUP em 181 crianças menores de um ano em Embu das Artes, São Paulo, observando frequência de consumo de um ou mais AUP de $43,1 \%$, o que correspondeu a quase metade da observada no presente estudo. Essa menor ocorrência pode estar relacionada ao uso de formulário com lista de alimentos restrita, o que poderia ter subestimado o consumo de AUP, enquanto no presente estudo todos os AUP consumidos no dia anterior à entrevista foram registrados com o uso de uma pergunta aberta sobre consumo de outros alimentos não incluídos na lista padronizada.

Nesta pesquisa, crianças de famílias com índice de riqueza abaixo da média apresentaram maior frequência de consumo de AUP. Na mesma linha, Martins e colaboradores ${ }^{24}$ identificaram contribuição crescente do consumo de AUP na dieta brasileira, principalmente em estratos da população de baixa renda. Além disso, Momm e Hofelmann ${ }^{25}$, em estudo realizado com crianças em escola municipal de Itajaí, Santa Catarina $(\mathrm{n}=523)$, observaram $52,6 \%$ de inadequação na dieta a partir de questionário elaborado com base no Guia alimentar para a população brasileira. Os autores concluíram que a alimentação de melhor qualidade foi diretamente associada a maiores estratos de renda familiar e melhores níveis de escolaridade. Ainda nesse sentido, Keane et $a l .{ }^{26}$ e Kendzor et al..$^{27}$, em estudos conduzidos em países de alta renda, apontaram que o baixo status socioeconômico e a baixa escolaridade são fatores que podem contribuir para o sobrepeso e a obesidade infantil. Esses achados corroboram estudo de revisão sistemática que aponta associação positiva entre consumo de AUP e gordura corporal durante a infância e a adolescência ${ }^{6}$.

A associação entre maior consumo de AUP e menor nível de escolaridade materna identificada neste estudo confirma os achados de Saldiva e colaboradores ${ }^{28}$ de que, a partir de dados obtidos da 2a Pesquisa de Prevalência de Aleitamento Materno conduzida nas Capitais Brasileiras e Distrito Federal em 2009, crianças com mães de menores níveis de escolaridade possuem frequência de consumo de AUP cerca de duas a três vezes maior, quando comparadas com mães de maiores níveis de escolaridade em análises brutas. Do mesmo modo, Relvas e colaboradores ${ }^{12}$ encontraram maior consumo de AUP em crianças filhas de mães com menor escolaridade.

Verificou-se, no presente estudo, que filhos de mães adolescentes apresentaram maior prevalência de consumo de AUP quando comparados aos filhos de mulheres adultas. De forma similar, estudo transversal $(n=112)$ realizado em Recife, avaliando o perfil alimentar materno e as práticas da alimentação complementar de crianças 
Tabela 2. Análise bruta das exposições socioeconômicas, demográficas, obstétricas e características da criança ao consumo de três ou mais alimentos ultraprocessados (AUP) no primeiro ano de vida $(n=774)$. Coorte MINABrasil, Cruzeiro do Sul, Acre, 2016-2017.

\begin{tabular}{|c|c|c|c|}
\hline \multirow{2}{*}{ Variáveis } & \multicolumn{2}{|c|}{ Modelo Bruto } & \multirow[b]{2}{*}{$\mathbf{p}^{\mathbf{e}}$} \\
\hline & $\mathbf{R P}$ & IC $95 \%$ & \\
\hline \multicolumn{4}{|l|}{ Idade materna } \\
\hline$\geq 19$ anos & & Ref. & \\
\hline$<19$ anos & 1,51 & 1,$26 ; 1,82$ & $<0,001$ \\
\hline \multicolumn{4}{|l|}{ Escolaridade materna } \\
\hline$>12$ anos & & Ref. & \\
\hline 10 a 12 anos & 1,74 & 1,$26 ; 2,40$ & 0,001 \\
\hline$\leq 9$ anos & 2,38 & 1,$73 ; 3,28$ & $<0,001$ \\
\hline \multicolumn{4}{|l|}{ Índice de riqueza } \\
\hline Acima da média & & Ref. & \\
\hline Abaixo da média & 1,48 & 1,$24 ; 1,77$ & $<0,001$ \\
\hline \multicolumn{4}{|l|}{ Bolsa Família } \\
\hline Não recebe & & Ref. & \\
\hline Recebe & 1,25 & 1,$05 ; 1,49$ & 0,011 \\
\hline \multicolumn{4}{|l|}{ Cor da pele materna } \\
\hline Branca & & Ref. & \\
\hline Negra/indígena/parda & 1,08 & 0,$82 ; 1,43$ & 0,567 \\
\hline \multicolumn{4}{|l|}{ Mãe exerce atividade remunerada } \\
\hline Sim & & Ref. & \\
\hline Não & 1,49 & 1,$22 ; 1,84$ & $<0,001$ \\
\hline \multicolumn{4}{|l|}{ Vive com companheiro } \\
\hline Sim & & Ref. & \\
\hline Não & 1,15 & 0,$95 ; 1,40$ & 0,164 \\
\hline \multicolumn{4}{|l|}{ Mãe chefe do domicílio } \\
\hline Não & & Ref. & \\
\hline Sim & 0,81 & 0,$61 ; 1,08$ & 0,150 \\
\hline \multicolumn{4}{|l|}{ Primigesta } \\
\hline Sim & & Ref. & \\
\hline Não & 0,87 & 0,$73 ; 1,03$ & 0,103 \\
\hline \multicolumn{4}{|l|}{ Número de consultas pré-natal } \\
\hline$\geq 6$ consultas & & Ref. & \\
\hline$<6$ consultas & 1,14 & 0,$93 ; 1,40$ & 0,220 \\
\hline \multicolumn{4}{|l|}{ Estado nutricional pré-gestacional } \\
\hline Peso adequado & & Ref. & \\
\hline Baixo peso & 1,21 & 0,$86 ; 1,70$ & 0,284 \\
\hline Sobrepeso & 0,82 & 0,$66 ; 1,00$ & 0,052 \\
\hline Obesidade & 0,79 & 0,$59 ; 1,04$ & 0,096 \\
\hline \multicolumn{4}{|l|}{ Peso ao nascer (gramas) } \\
\hline Adequado (2.500 a 3.999) & & Ref. & \\
\hline Baixo peso $(<2.500)$ & 0,90 & 0,$61 ; 1,31$ & 0,564 \\
\hline Macrossomia $(\geq 4.000)$ & 0,88 & 0,$60 ; 1,30$ & 0,524 \\
\hline \multicolumn{4}{|l|}{ Aleitamento materno no $1^{\circ}$ dia } \\
\hline $\operatorname{Sim}$ & & Ref. & \\
\hline Não & 0,87 & 0,$54 ; 1,41$ & 0,581 \\
\hline \multicolumn{4}{|l|}{ Uso de mamadeira ${ }^{\mathrm{a}}$} \\
\hline Não & & Ref. & \\
\hline Sim & 0,92 & 0,$77 ; 1,10$ & 0,351 \\
\hline
\end{tabular}


Tabela 2. Análise bruta das exposições socioeconômicas, demográficas, obstétricas e características da criança ao consumo de três ou mais alimentos ultraprocessados (AUP) no primeiro ano de vida $(\mathrm{n}=774)$. Coorte MINABrasil, Cruzeiro do Sul, Acre, 2016-2017.

\begin{tabular}{|c|c|c|c|}
\hline \multirow{2}{*}{ Variáveis } & \multicolumn{2}{|c|}{ Modelo Bruto } & \multirow[b]{2}{*}{$\mathbf{p}^{\mathrm{e}}$} \\
\hline & RP & IC 95\% & \\
\hline \multicolumn{4}{|l|}{ Uso de chupeta ${ }^{\mathrm{a}}$} \\
\hline Não & & Ref. & \\
\hline Sim & 0,93 & 0,$75 ; 1,15$ & 0,513 \\
\hline \multicolumn{4}{|c|}{$\begin{array}{l}\text { Aleitamento materno exclusivo e predominante até } 6 \\
\text { meses }(n=628)\end{array}$} \\
\hline Sim & & Ref. & \\
\hline Não & 0,76 & 0,$57 ; 1,02$ & 0,064 \\
\hline \multicolumn{4}{|l|}{ Aleitamento materno aos 12 meses } \\
\hline Sim & & Ref. & \\
\hline Não & 1,00 & 0,$83 ; 1,21$ & 0,980 \\
\hline \multicolumn{4}{|l|}{ Déficit de comprimento para idade ${ }^{\mathrm{a}, \mathrm{b}}$} \\
\hline Não & & Ref. & \\
\hline $\operatorname{Sim}$ & 1,47 & 0,$98 ; 2,21$ & 0,064 \\
\hline \multicolumn{4}{|l|}{ Excesso de peso ${ }^{\mathrm{a}, \mathrm{c}}$} \\
\hline Não & & Ref. & \\
\hline Sim & 0,67 & 0,$42 ; 1,05$ & 0,078 \\
\hline \multicolumn{4}{|l|}{ Diversidade da dieta ${ }^{a, d}$} \\
\hline Sim & & Ref. & \\
\hline Não & 0,71 & 0,$56 ; 0,90$ & 0,005 \\
\hline
\end{tabular}

Fonte: Autoras.

com idades entre 9 e 24 meses, encontrou maior consumo de alimentos ricos em açúcar, óleos e gorduras em filhos de mães adolescentes, quando comparados aos filhos de mães adultas ${ }^{29}$. Esse fato pode estar relacionado à tendência de o público mais jovem ter, cada vez mais, práticas alimentares que correspondem a dietas ricas em sódio, gordura e açúcares, porém carentes em hortaliças $^{30}$. Estudo transversal realizado em Teresina, Piauí, avaliou o consumo alimentar de 80 jovens segundo a classificação de alimentos NOVA, que categoriza os alimentos de acordo com a extensão e o propósito de seu processamento. No total, 94\% dos adolescentes consumiam AUP uma ou mais vezes ao $\mathrm{dia}^{31}$.

Como limitação do presente estudo, deve ser considerado que foram avaliados apenas indivíduos residentes da zona urbana e peri-urbana de Cruzeiro do Sul, uma vez que há dificuldade de localização e acesso à zona rural da cidade, portanto os dados não podem ser generalizados para toda a população do município. Embora esta pesquisa seja de base populacional, perdemos o seguimento de alguns participantes, sendo maior o número de perdas de crianças nascidas de mães mais jovens, com menor escolaridade e mais pobres. Por outro lado, destaca-se que os resultados foram baseados em dados coletados prospectivamente em uma área pouco estudada, com procedimentos contínuos para verificação da consistência dos dados e constante treinamento da equipe de pesquisa.

Quanto ao consumo de AUP, utilizou-se questionário relativo à ingestão do dia anterior à entrevista, o que minimiza a possibilidade de viés de memória materna, melhorando a qualidade dos dados apresentados. Outra limitação foi a avaliação de apenas um dia de consumo alimentar, o que não representa o consumo habitual. Com o intuito de minimizar essa limitação, os avaliadores se atentaram em confirmar com as mães ou cuidadores se a alimentação do dia anterior teria sido a habitual da criança. Para 5\% das crianças estudadas, o dia anterior à entrevista não representava um dia de alimentação habitual. Nesses casos, foi registrado o consumo do último dia habitual da criança.

O presente estudo revela um nível de consumo elevado de AUP em crianças no primeiro ano de vida em Cruzeiro do Sul, ressaltando a neces- 
Tabela 3. Razão de Prevalência e intervalo com 95\% de confiança (IC 95\%) para fatores associados ao consumo de três ou mais alimentos ultraprocessados (AUP) no primeiro ano de vida $(\mathrm{n}=774)$. Coorte MINA-Brasil, Cruzeiro do Sul, Acre, $2016-2017$.

\begin{tabular}{|c|c|c|c|c|c|c|}
\hline \multirow{3}{*}{ Variáveis } & \multicolumn{6}{|c|}{ Consumo de três ou mais AUP no dia anterior à entrevista $(n=774)$} \\
\hline & \multicolumn{2}{|c|}{ Modelo bruto } & \multicolumn{2}{|c|}{ Modelo $1^{\mathrm{a}}$} & \multicolumn{2}{|l|}{ Modelo $2^{\text {b }}$} \\
\hline & RP (IC 95\%) & $\mathbf{p}$ & RP (IC 95\%) & $\mathbf{p}$ & RP (IC 95\%) & $\mathbf{p}$ \\
\hline \multicolumn{7}{|l|}{ Idade materna } \\
\hline$\geq 19$ anos & Ref. & & Ref. & & Ref. & \\
\hline$<19$ anos & $1,51(1,26 ; 1,82)$ & $<0,001$ & $1,21(0,98 ; 1,48)$ & 0,073 & $1,28(1,06 ; 1,55)$ & 0,010 \\
\hline \multicolumn{7}{|l|}{ Escolaridade materna } \\
\hline$>12$ anos & Ref. & & Ref. & & Ref. & \\
\hline $10-12$ anos & $1,74(1,26 ; 2,40)$ & 0,001 & $1,51(1,07 ; 2,12)$ & 0,020 & $1,58(1,13 ; 2,20)$ & 0,007 \\
\hline$\leq 9$ anos & $2,38(1,73 ; 3,28)$ & $<0,001$ & $1,89(1,31 ; 2,74)$ & $<0,001$ & $1,97(1,38 ; 2,80)$ & $<0,001$ \\
\hline \multicolumn{7}{|l|}{ Índice de riqueza } \\
\hline Acima da média & Ref. & & Ref. & & Ref. & \\
\hline Abaixo da média & $1,48(1,24 ; 1,77)$ & $<0,001$ & $1,20(0,99 ; 1,46)$ & 0,068 & $1,26(1,04 ; 1,53)$ & 0,018 \\
\hline \multicolumn{7}{|l|}{ Bolsa Família } \\
\hline Não recebe & Ref. & & Ref. & & & \\
\hline Recebe & $1,25(1,05 ; 1,49)$ & 0,011 & $0,97(0,81 ; 1,16)$ & 0,743 & & \\
\hline \multicolumn{7}{|l|}{ Mãe chefe domicílio } \\
\hline Não & Ref. & & Ref. & & & \\
\hline Sim & $0,81(0,61 ; 1,08)$ & 0,150 & $0,84(0,63 ; 1,11)$ & 0,217 & & \\
\hline \multicolumn{7}{|c|}{ Mãe exerce atividade remunerada } \\
\hline Sim & Ref. & & Ref. & & & \\
\hline Não & $1,49(1,22 ; 1,84)$ & $<0,001$ & $1,11(0,88 ; 1,39)$ & 0,377 & & \\
\hline \multicolumn{7}{|l|}{ Vive com companheiro } \\
\hline Sim & Ref. & & Ref. & & & \\
\hline Não & $1,15(0,95 ; 1,40)$ & 0,164 & $1,07(0,87 ; 1,31)$ & 0,551 & & \\
\hline \multicolumn{7}{|c|}{ Estado nutricional pré-gestacional } \\
\hline Peso adequado & Ref. & & Ref. & & Ref. & \\
\hline Baixo peso & $1,21(0,86 ; 1,70)$ & 0,284 & $1,15(0,81 ; 1,63)$ & 0,452 & $1,16(0,82 ; 1,65)$ & 0,401 \\
\hline Sobrepeso & $0,82(0,66 ; 1,00)$ & 0,052 & $0,85(0,70 ; 1,05)$ & 0,128 & $0,85(0,69 ; 1,04)$ & 0,104 \\
\hline Obesidade & $0,79(0,59 ; 1,04)$ & 0,096 & $0,81(0,61 ; 1,07)$ & 0,138 & $0,80(0,60 ; 1,06)$ & 0,117 \\
\hline \multicolumn{7}{|l|}{ Primigesta } \\
\hline Sim & Ref. & & Ref. & & & \\
\hline Não & $0,87(0,73 ; 1,03)$ & 0,103 & $0,91(0,74 ; 1,11)$ & 0,357 & & \\
\hline
\end{tabular}

Aleitamento materno exclusivo ou predominante até 6 meses

Sim

Não

Déficit de comprimento para idade

Não

$\operatorname{Sim}$

Excesso de peso ${ }^{c}$

Não

$\operatorname{Sim}$

Diversidade da dieta ${ }^{c, d}$

Sim

Ref.

$0,76(0,57 ; 1,02)$

Ref.

$1,47(0,98 ; 2,21)$

Ref.

$0,67(0,42 ; 1,05)$

Ref.
Ref. 0,064
Ref. $0,78(0,58 ; 1,03)$

Ref. 0,064 $1,20(0,74 ; 1,94)$

Ref. $0,73(0,46 ; 1,16) \quad 0.184$

Não

Ref.

Ref. $0,71(0,56 ; 0,90) \quad 0,005 \quad 0,65(0,52 ; 0,81) \quad<0,001 \quad 0,65(0,51 ; 0,81) \quad<0,001$ ${ }^{a}$ Modelo 1: idade materna (<19 anos, $\geq 19$ anos), escolaridade ( $\leq 9$ anos, 10 a 12 anos, $>12$ anos), índice de riqueza (abaixo da média, acima da média), recebimento do auxílio Bolsa Família (sim, não), mãe chefe do domicílio (sim, não), mãe exerce atividade remunerada (sim, não), viver com companheiro (sim, não), estado nutricional pré-gestacional (baixo peso, peso adequado, sobrepeso e obesidade), primigesta (sim, não), aleitamento materno exclusivo ou predominante aos seis meses de vida (sim, não), déficit de comprimento para idade no seguimento de um ano (sim, não), excesso de peso no seguimento de um ano (sim, não) e diversidade da dieta no seguimento de um ano (sim, não), com ajuste para idade e sexo da criança. ${ }^{b}$ Modelo 2: modelo 1, sem ajuste para Bolsa Família, mãe chefe do domicílio, mãe exerce atividade remunerada, viver com companheiro, primigesta, déficit de comprimento para idade, excesso de peso. ${ }^{\mathrm{c}}$ No seguimento de um ano da coorte. ${ }^{\mathrm{d}}$ Calculado segundo indicador de diversidade da alimentação complementar proposto pela OMS $(2008)^{22}$.

Fonte: Autoras. 
sidade de ações para promoção da alimentação adequada nessa fase da vida. Cabe aos profissionais de saúde, gerentes e formuladores de políticas de saúde e nutrição enfrentar esse grande desafio. Recomenda-se a ampliação da divulgação do Guia alimentar brasileiro para menores de dois anos entre famílias e profissionais de saúde, a realização de intervenções educativas em ambientes de atenção primária à saúde, visando melhorar as práticas de alimentação infantil, e o monitoramento contínuo das práticas de alimentação na primeira infância, com base nas recomendações do Guia alimentar para crianças brasileiras.

Conhecer a situação da alimentação complementar no local é essencial para a aplicação de iniciativas já idealizadas e para a definição de políticas e programas de intervenção precoce que visem a promoção da saúde infantil, bem como o aperfeiçoamento e a maior divulgação de leis e regulamentações brasileiras para propagandas e comercialização de alimentos destinados a crianças, tais como a NBCAL - Norma Brasileira para Comercialização de Alimentos para Lácteos e Crianças de Primeira Infância, Bicos, Chupetas e Protetores de Mamilos. Os resultados deste estudo contribuem com evidências que reforçam a importância do incentivo a políticas públicas que desestimulem o consumo desse tipo de alimento, sobretudo entre os grupos de maior vulnerabilidade social.

\section{Conclusão}

O presente estudo apontou um alto consumo de AUP no seguimento de um ano de vida da coorte, dada a recomendação do Guia alimentar brasileiro de que não sejam ofertados AUP nos dois primeiros anos de vida. O consumo de três ou mais AUP no dia anterior à entrevista foi associado a características maternas de menores escolaridade, índice de riqueza e idade materna. A diversidade da dieta foi associada ao maior consumo de AUP, alertando para a necessidade de incluir a avaliação do consumo de AUP como um indicador de alimentação complementar, conforme recomendações do Guia alimentar brasileiro para crianças menores de dois anos, visando o monitoramento apropriado das práticas de alimentação infantil, além do desenvolvimento e implantação de estratégias sólidas para promoção da alimentação saudável desde a gestação, desencorajando a oferta de AUP nos primeiros dois anos de vida.

\section{Colaboradores}

MB Nogueira trabalhou na análise e interpretação dos dados, elaboração e revisão do manuscrito. L Mazzucchetti contribuiu na análise dos dados e revisão crítica do manuscrito. OS Mosquera atuou na coleta dos dados, interpretação e revisão crítica do manuscrito. MA Cardoso trabalhou na concepção e delineamento do estudo, coleta dos dados e revisão crítica do manuscrito. MB Malta atuou no planejamento do estudo, coleta, análise e interpretação dos dados e na revisão crítica do manuscrito. 
7. Pan American Health Organization (PAHO). Ultra -processed food and drink products in Latin America: trends, impact on obesity, policy implications. Washington, DC: PAHO; 2015.

8. Levy RB, Claro RM, Mondini L, Sichieri R, Monteiro CA. Distribuição regional e socioeconômica da disponibilidade domiciliar de alimentos no Brasil em 20082009. Rev Saude Publica. 2012; 46(1):6-15.

9. Longo-Silva G, Silveira JAC, Menezes RCE, Toloni MHA. Age at introduction of ultra-processed food among preschool children attending day-care centers. J Pediatr 2017; 93(5):508-516.

10. Brasil. Ministério da Saúde (MS). Secretaria de Atenção à Saúde. Departamento de ações Programáticas e Estratégicas. II Pesquisa de Prevalência de Aleitamento materno nas Capitais Brasileiras e Distrito Federal. Brasilia: MS; 2009.

11. Flores TR, Nunes BP, Neves RG, Wendt AT, Costa CS, Wehrmeister FC, Bertoldi AD. Consumo de leite materno e fatores associados em crianças menores de dois anos: Pesquisa nacional de saúde, 2013. Cad Saude Publica 2017; 33(11):e00068816.

12. Relvas GRB, Buccini GS, Venancio SI. Ultra-processed food consumption among infants in primary health care in a city of the metropolitan region of Sao Paulo, Brazil. J Pediatr (Rio J) 2019; 95(5)584-589.

13. Cardoso M, Matijasevich A, Malta MB, Lourenco BH, Gimeno SGA, Ferreira MU, Castro MC. Cohort profile: The Maternal and Child Health and Nutrition in Acre, Brazil, birth cohort study (MINA-Brazil). BMJ Open 2020; 10(2):e034513.

14. De Onis M. WHO Child Growth Standards based on length/height, weight and age. Acta Paediatr 2006; 95:76-85.

15. Filmer D, Pritchett LH. Estimating wealth effects without expenditure data - or tears: an application to educational enrollments in states of India. Demography 2001; 38(1):115-132.

16. Brasil. Ministério da Saúde (MS). Portaria n 569. Programa de Humanização no Pré-natal e Nascimento. Diário Oficial da União 2000; 1 jun.

17. Dal Bom JP, Mazzucchetti L, Malta MB, Ladeia-Andrade S, De Castro MC, Cardoso MA, Lourenço BA. Early determinants of linear growth and weight attained in the first year of life in a malaria endemic region. PLoS One 2019; 14(8):e0220513.

18. Oliveira JM, Castro IRR, Silva GB, Venancio SI, Saldiva SRDM. Avaliação da alimentação complementar nos dois primeiros anos de vida: proposta de indicadores e de instrumento. Cad Saude Publica 2015; 31(2):377-394.

19. World Health Organization (WHO). Physical status: the use and interpretation of report anthropometry. Report of a WHO Expert Committee. Geneva: WHO; 1995.

20. Castro TG, Baraldi LG, Muniz PT, Cardoso MA. Dietary practices and nutritional status of 0-24-month -old children from Brazilian Amazonia. Public Health Nutr 2009; 12(12):2335-2342.

21. World Health Organization (WHO). Indicators for assessing infant and young child feeding practices. Part 1: definitions. Washington DC: WHO; 2008.
22. Victora CG, Huttly SR, Fuchs SC, Olinto MTA. The role of conceptual frameworks in epidemiological analysis: a hierarchical approach. Int J Epidemiol 1997; 26(1):224-227.

23. Victora CG, Horta BL, Loret de Mola C, Quevedo L, Pinheiro RT, Gigante DP, Gonçalves H, Barros FC. Association between breastfeeding and intelligence, educational attainment, and income at 30 years of age: a prospective birth cohort study from Brazil. Lancet Glob Health 2015; 3(4):e199-205.

24. Martins APB, Levy RB, Claro RM, Moubarac JC, Monteiro CA. Increased contribution of ultra-processed food products in the Brazilian diet (1987-2009). Rev Saude Publica 2013; 47(4):656-665.

25. Momm N, Höfelmann DA. Qualidade da dieta e fatores associados em crianças matriculadas em uma escola municipal de Itajaí, Santa Catarina. Cad Saude Colet 2014; 22(1):32-39.

26. Keane E, Layte R, Harrington J, Kearney PM, Perry IJ. Measured parental weight status and familial socio-economic status correlates with childhood overweight and obesity at age 9. PLoS One 2012; 7(8):e43503.

27. Kendzor DE, Caughy MO, Owen MT. Family income trajectory during childhood is associated with adiposity in adolescence: a latent class growth analysis. BMC Public Health 2012; 12:611.

28. Saldiva SR, Venancio SI, Santana AC, Silva Castro AL, Escuder MM, Giugliani ER. The consumption of unhealthy foods by Brazilian children is influenced by their mother's educational level. Nutr J 2014. 13:33.

29. Costa KAO, Antunes MMC, Cabral PC, Silva GAP. Feeding style of adolescent mothers and complementary feeding practice of their infants. Rev Nutr 2018; 31(1):49-58.

30. Toral N, Conti MA, Slater E. A alimentação saudável na ótica dos adolescentes : percepções e barreiras à sua implementação e características esperadas em materiais educativos. Cad Saude Publica 2009; 25(11):2386-2394.

31. Coelho BMS, Macedo MAD, Pereira TG. Avaliação do consumo alimentar de adolescentes segundo a nova classificação de alimentos - NOVA. Revista Interdisciplinar 2017; 10(2):32-39

Artigo apresentado em 24/09/2020

Aprovado em 26/01/2021

Versão final apresentada em 28/01/2021

Editores-chefes: Romeu Gomes, Antônio Augusto Moura da Silva 